\title{
A MAXIMAL RIEMANN SURFACE
}

\author{
MARTIN JURCHESCU
}

We let the notations be as in [3]. Then, in the category 6 of all bordered Riemann surfaces, the following inclusion diagram holds [3, Theorem 9]:

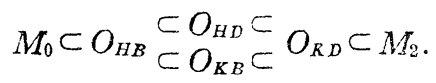

Further, from a theorem of Kuramochi [4] (see also Constantinescu and Cornea [2]), it easily follows that the class $O_{A D}$ is not contained in $M_{2}$. On the other hand, it is well known that $M_{2}$ (which equals $O_{S B}$ for ordinary planar surfaces) is not contained in $O_{A D}$ (see Ahlfors and Beurling [1]).

Now let $\xi_{0}$ be the subcategory of bordered Riemann surfaces without planar ideal boundary. Then $\dot{F}_{0} \cap M_{2}=M=$ the class of all maximal bordered Riemann surfaces. Hence the question whether $M$ is or not contained in $O_{A D}$ naturally arises; it was first considered by Sario [j]. This note contains the negative answer to Sario's question.

Let $X=R \cup B$ and $X_{0}=R_{0} \cup B_{0}$ be two bordered Riemann surfaces. We recall that a continuous map $f: X \rightarrow X_{0}$ is said to be distinguished if $f(B) \subset B_{0}$, and proper if, for any compact $K_{0} \subset X_{0}, f^{-1}\left(K_{0}\right)$ is compact. Let $M_{1}$ be the class of all bordered Riemann surfaces with absolutely disconnected ideal boundary.

TheоRem 1. Suppose there exists a distinguished proper conformal map $f: X \rightarrow X_{0}$. Then $X \in M_{1}$ if and only if $X_{0} \in M_{1}$.

Proof. Let $\beta$ and $\beta_{0}$ be the nowhere disconnecting and 0-dimensional ideal boundaries of $X$ and $X_{0}$. Then the spaces $X^{*}=X \cup \beta$ and $X_{0}^{*}=X_{0} \cup \beta_{0}$ are compact and locally connected, and the sets $\beta$ and $\beta_{0}$ are nowhere disconnecting and 0 -dimensional. By Lemma 2 in [3], the proper map $f: X \rightarrow X_{0}$ can be extended to a continuous map $f^{*}: X^{*} \rightarrow X_{0}^{*}$ satisfying $f^{*}(\beta)=\beta_{0}$ and $f^{*-1}\left(\beta_{0}\right)$ $=\beta$.

Received June 26, 1961. 
For any $x \in X$, let $o(x)$ denote the multiplicity of $f$ at $x$. As $f$ is conformal, proper and distinguished, there exists a natural number $s$ such that

$$
\sum_{x \in f^{-1}\left(\infty_{0}\right)} O(x)=s,
$$

for any $x_{0} \in X_{0}[6, \mathrm{p} .126]$. Let $E$ be the set of all points $x \in X$ for which $o(x)>1$. Then $E$ is discrete in $X$, and $E_{0}=f(E)$ is discrete in $X_{0}$.

Choose, in a parametric neighborhood on $X_{0}$, a disc $\bar{U}_{0}$ which does not meet $E_{0}$, and let $\bar{U}=f^{-1}\left(\bar{U}_{0}\right)$. Then $Y_{0}=X_{0}-\bar{U}_{0}$ is a normal neighborhood [3, Definition 8] in $X_{0}$ of $\beta_{0}$, and $Y=X-\bar{U}$ is a normal neighborhood in $X$ of $\beta$. Let $\left(Y_{0, n}\right)_{n \in N}$ be a relative exhaustion $[3$, Definition 7$]$ of $Y_{0}$ such that $\beta_{0, n}$ does not meet $E_{0}$ for any $n \in N$, where $\beta_{0, n}=\partial Y_{0, n}-\partial Y_{0}$ and where $\partial$ stands for the relative boundary. Then $\left(Y_{n}\right)_{n \in N}$ is a relative exhaustion of $Y$, where $Y_{n}=f^{-1}\left(Y_{0, n}\right)$. Let $\beta_{n}=f^{-1}\left(\beta_{0, n}\right)=\partial Y_{n}-\partial Y$. Let $\alpha_{0}$ be a subset of $\beta_{0}, \alpha$ $=f^{*-1}\left(\alpha_{0}\right), \mu_{\alpha_{0}}$ the modulus of $Y_{0}$ for $\partial Y_{0}$ and $\alpha_{0}$ and $\mu_{\varepsilon}$ the modulus of $Y$ for $\partial Y$ and $\alpha[3$, Definition 13]. It will be proved that

$$
\mu_{a}=\frac{1}{s} \mu_{a_{0}}
$$

Let $\alpha_{0, n}$ be the minimal subcycle of $\beta_{0, n}$ which separates $\alpha_{0}$ from $\partial Y$. Then, since $f^{*}$ is continuous, $\alpha_{n}=f^{-1}\left(\alpha_{0, n}\right)$ is the minimal subcycle of $\beta_{n}$ which separates $\alpha$ from $\partial Y$. Let $u_{0, n}$ and $\mu_{0, n}$ be the extremal function and the modulus of $Y_{0, n}$ for $\partial Y_{0}$ and $\alpha_{0, n}$, and let $u_{n}$ and $\mu_{n}$ be the extremal function and the modulus of $Y_{n}$ for $\partial Y$ and $\alpha_{n}$. By Lemma 8 in [3], we have

$$
u_{n}=\frac{1}{s} u_{0, n} \circ f
$$

Hence $\mu_{n}=\frac{1}{s} \mu_{0, n}$ and so, as $n \rightarrow \infty$,

$$
\mu_{\alpha}=\frac{1}{s} \mu_{\alpha_{0}}
$$

as asserted. From this equality it follows that $\alpha_{0}$ is parabolic [3, Definition 14] if and only if $\alpha$ is parabolic. In particular, $\gamma_{0} \in \beta_{0}$ is parabolic if and only if $f^{*-1}\left(\gamma_{0}\right)$ is parabolic. But it is easily seen that the set $f^{*-1}\left(\gamma_{0}\right)$ is finite. Thus $r_{0}$ is parabolic if and only if all $\gamma \in f^{*-1}\left(\gamma_{0}\right)$ are parabolic [3, Corollary 4]. The theorem now follows. 
Remark. An immediate corollary of Theorem 1 is the following statement:

If $X_{0}$ is relatively planar and $X_{0} \in O_{B B}$ and if there exists a distinguished proper conformal map $f: X \rightarrow X_{0}$, then $X$ is essentially maximal.

A direct proof of this statement, in the ordinary case, was given by Tamura [7].

TheOREM 2. There exists a maximal ordinary Riemann surface $X \notin O_{A D}$.

Proof. According to Ahlfors and Beurling [1, Theorem 16], there exists a planar ordinary Riemann surface $X_{0} \in O_{S B}-O_{A D}$. As $M_{1}=O_{S B}$ for planar ordinary surfaces, this $X_{0}$ belongs to $M_{1}-O_{A D}$.

Let $E_{0}$ be a discrete subset of $X_{0}$ having the property that the closure in $X_{0}^{*}$ of $E_{0}$ is $E_{0} \cup \beta_{0}$. Then there exists an ordinary Riemann surface $X$ and a proper conformal map $f: X \rightarrow X_{0}$ such that $f^{-1}\left(x_{0}\right)$ contains a single point for any $x_{0} \in E_{0}$, and such that $o(x)=2$ if $x \in f^{-1}\left(E_{0}\right)$ and $o(x)=1$ if $x \in X-f^{-1}\left(E_{0}\right)$.

It is clear that $X$ has no boundary components of planar type. As $X_{0} \in M_{1}$, $X \in M_{1}$ by Theorem 1 , and consequently $X$ is essentially maximal. As $X_{0} \notin O_{A D}$, it is easily seen that $X \notin O_{A D}$. Thus the proof is complete.

\section{REFERENCES}

[1] Ahlfors, L. and A. Beurling: Conformal invariants and function-theoretic null sets, Acta Math. 83, 101-129 (1950).

[2] Constantinescu C. and A. Cornea: Über den idealen Rand und einige seiner Anwendungen bei der Klassifikation der Riemannschen Flächen, Nagoya Math. J. 13, 169-233 (1958).

[ 3 ] Jurchescu, M.: Bordered Riemann surfaces, Math. Ann. 143, 264-292 (1961).

[4] Kuramochi, Z.: On the behaviour of analytic functions on abstract Riemann surfaces, Osaka Math. J. 7, 109-127 (1955).

[5] Sario, L.: Über Riemannsche Flächen mit hebbarem Rand, Ann. Acad. Sci. Fenn. Ser. A. I. 50, 1-79 (1948).

[6] Stoïlow, S: Leçons sur les principes topologiques de la théorie des fonctions analytiques, Paris, Gauthier-Villars (2nd ed) (1956).

[7] Tamura, J.: On a theorem of Tsuji, Japanese J. Math. 29, 138-140 (1959).

Rumanian Academy 